www4.fsanet.com.br/revista

Rev. FSA, Teresina, v. 18, n. 02, art. 10, p. 183-197, fev. 2021

ISSN Impresso: 1806-6356 ISSN Eletrônico: 2317-2983 http://dx.doi.org/10.12819/2021.18.02.10

\title{
Gamificação como Estratégia para Estimular a Aprendizagem dos Alunos na Escola: Uma Análise Bibliométrica
}

Gamification as a Strategy to Stimulate Student Learning at School: A Bibliometric Analysis

Cleide Ane Barbosa da Cruz

Doutora em Ciência da Propriedade Intelectual pela Universidade Federal de Sergipe Professor do Instituto Federal de Sergipe - Campus Tobias Barreto

E-mail: cleideane.barbosa@bol.com.br

Vinicius Marques Nejaim

Doutorando em Ciência da Propriedade Intelectual pela Universidade Federal de Sergipe Professor do Instituto Federal de Sergipe - Campus Itabaiana

E-mail: vinicius.nejaim@ifs.edu.br

Aline Santos Soares Bezerra Professor do Instituto Federal de Sergipe - Campus Aracaju

E-mail: aline.bezerra@ifs.edu.br

Endereço: Cleide Ane Barbosa da Cruz

Instituto Federal de Sergipe - Campus Tobias Barreto -

Rod. Antônio Carlos Valadares, s\n, Tobias Barreto,

CEP: 49300000 - Tobias Barreto, SE - Brasil.

Endereço: Vinicius Marques Nejaim

Instituto Federal de Sergipe - Campus Tobias Barreto Rod. Antônio Carlos Valadares, s\n, Tobias Barreto, CEP: 49300000 - Tobias Barreto, SE - Brasil.

Endereço: Aline Santos Soares Bezerra

Av. Eng. Gentil Tavares, 1166 - Getúlio Vargas, 49055260 - Aracaju - SE - Brasil.
Editor-Chefe: Dr. Tonny Kerley de Alencar Rodrigues

Artigo recebido em 04/01/2020. Última versão recebida em 21/01/2021. Aprovado em 22/01/2021.

Avaliado pelo sistema Triple Review: a) Desk Review pelo Editor-Chefe; e b) Double Blind Review (avaliação cega por dois avaliadores da área).

Revisão: Gramatical, Normativa e de Formatação 


\title{
RESUMO
}

Com o passar dos anos, as tecnologias da informação e comunicação passaram a ser inseridas nas escolas, e com isso a gamificação surgiu como forma de melhorar o ensino e aprendizagem dos alunos, sendo que já existem produções científicas que tratam da aplicação da gamificação na educação. Por isso, esta pesquisa tem como objetivo apresentar uma análise bibliométrica de publicações científicas sobre gamificação aplicada à educação. Em relação à metodologia, este estudo se classifica como exploratório quantitativo. Foi realizada uma análise bibliométrica por meio de uma busca na base Scopus, sendo encontradas 210 publicações entre os anos de 2013 a 2019. Os resultados evidenciam que as produções científicas voltadas à aplicação da gamificação na educação são recentes, pois foram encontrados dados a partir de 2013. Além disso, constatou-se que a Espanha foi o país onde há uma concentração maior de publicações relacionadas ao assunto em estudo. A utilização do programa VOSviewer identificou palavras-chaves que correspondem a aplicação da gamificação, evidenciando que sua utilização é necessária para verificar se o que está sendo analisado envolve o objeto do estudo da pesquisa. Dessa forma, compreende-se que as publicações mostram que os estudos voltados à gamificação, aplicados à educação, vêm crescendo, porém, esses estudos são recentes, o que evidencia que ainda há muito a entender sobre sua aplicação e como essa metodologia pode melhorar a qualidade do ensino e aprendizagem dos alunos nas escolas.

Palavras-chaves: Educação. Jogos. Criatividade. Motivação. Cenários Lúdicos.

\begin{abstract}
Over the years, information and communication technologies started to be inserted in schools, and with that, gamification emerged as a way to improve the teaching and learning of students, and there are already scientific productions that deal with the application of gamification in education. Therefore, this research aims to present a bibliometric analysis of scientific publications on gamification applied to education. Regarding the methodology, this study is classified as quantitative exploratory. A bibliometric analysis was carried out through a search in the Scopus database, and 210 publications were found between the years 2013 to 2019. The results show that the scientific productions aimed at the application of gamification in education are recent, since data were found from 2013. In addition, it was found that Spain was the country where there is a greater concentration of publications related to the subject under study. The use of the VOSviewer program identified keywords that correspond to the gamification application, showing that its use is necessary to verify if what is being analyzed involves the object of the research study. Thus, it is understood that the publications show that the studies focused on gamification, applied to education, have been growing, however these studies are recent, which shows that there is still much to understand about its application and how this methodology can improve quality teaching and learning of students in schools.
\end{abstract}

Keywords: Education. Games. Creativity. Motivation. Playful Aettings. 


\section{INTRODUÇÃO}

Atualmente, as tecnologias da informação e comunicação (TIC), estão sendo inseridas no ambiente escolar, por meio de diferentes ferramentas e plataformas com recursos digitais. Isso se relaciona com o perfil dessa nova geração que joga vídeo game, o que permite que estas ferramentas possam ser introduzidas no ambiente de ensino e aprendizagem para estimular a motivação dos alunos (DUARTE, 2014).

Por isso, a gamificação surge como uma possível alternativa para atrair o interesse dos alunos, despertando sua curiosidade, associando elementos que estimulam a participação destes e o engajamento na escola, o que permite uma reinvenção do aprendizado (ORLANDI et al., 2018).

Além disso, esta prática vem ganhando espaço nos ambientes sociais, permitindo que educadores tenham maior êxito nos ambientes escolares, bem como ajudar empresas a alcançarem metas com mais rapidez. A tecnologia e a gamificação trabalham em conjunto com o desenvolvido de jogos que melhoram o desempenho de empresas e educadores (SALAMI et al., 2018).

A utilização de games no âmbito escolar é um recurso de aprendizagem que contribui para impulsionar a motivação dos alunos (LEFFA; PINTO, 2014), isso mostra que os elementos dos jogos contribuem para formação e construção de atividades que facilitem o desenvolvimento dos alunos no ambiente escolar.

Diante desta esta pesquisa, justifica-se pela necessidade de compreender o desenvolvimento das publicações científicas em relação à utilização da gamificação na educação, visto que se percebe o crescimento da utilização de mecanismos de jogos para melhoria do ensino e aprendizagem nas escolas, visando despertar a busca do conhecimento pelos alunos.

Para tanto, esta pesquisa utilizará a bibliometria que corresponde a uma metodologia de pesquisa das atividades científicas e correlatas, por intermédio de uma análise de dados, que permite, por exemplo, a identificação da quantidade de trabalhos sobre publicados sobre um específico assunto (KOBASHI SANTOS, 2008).

Dessa forma, esta pesquisa tem como objetivo apresentar uma análise bibliométrica de publicações científicas sobre gamificação aplicada à educação. 


\section{REFERENCIAL TEÓRICO}

\subsection{Conceitos de gamificação}

A gamificação abrange a utilização de mecanismos sistemáticos de jogos para a resolução de problemas e para a motivação e o engajamento de um determinado público. Seu objetivo é criar ou adaptar a experiência do usuário a um serviço, processo ou produto, permitindo que estes usuários explorem suas habilidades e propõem benefícios como recompensas físicas e virtuais as tarefas que forem realizadas (VIANNA et al., 2013).

O termo gamificação foi expressado pela primeira vez pelo pesquisador britânico Nick Pelling (VIANNA, 2013). Para Alves, Minho e Diniz (2014, pág. 76) “a gamificação se constitui na utilização da mecânica dos games emcenários non games, criando espaços de aprendizagem mediados pelo desafio, pelo prazer e entretenimento".

Por sua vez, este é um termo recente que surgiu do inglês "gamification", e é utilizando tanto por organizações como por educadores, sendo que pode ser usada em diversos ambientes, e não precisa ser digital, pode ser analógica, em sistemas de check-in, em metas atingidas, entre outros (SALAMI, 2018).

Entende-se que a gamificação não se limita a jogar um game, mas corresponde à aplicação de elementos e mecânica relacionados aos games em espaços e atividades não necessariamente aplicados aos games (ZICHERMANN; CUNNINGHAM, 2011). Em complemento, Vieira e Hoyos (2018) explicam que esta utiliza componentes próprios de um jogo, visando estimular a motivação da aprendizagem ou da realização de tarefas, que poderiam ser consideradas tarefas cansativas.

Ainda, esta também consiste na utilização de elementos de design de jogos no ambiente de aprendizagem visando impulsionar motivação e melhoria do desempenho dos alunos na escola (SILVA; SALES; CASTRO, 2019).

Para Rodrigues (2015) utilizar esses elementos significa realizar a seleção de características inerentes dos jogos, como por exemplo, pontuação, recompensa, emblemas, níveis de fases, e aplicar estes elementos em outros contextos, que não refiram-se a jogos. A seguir têm-se as variáveis que definem o conceito de Gamificação na Figura 1. 
Figura 1 - Variáveis que definem o conceito de gamificação

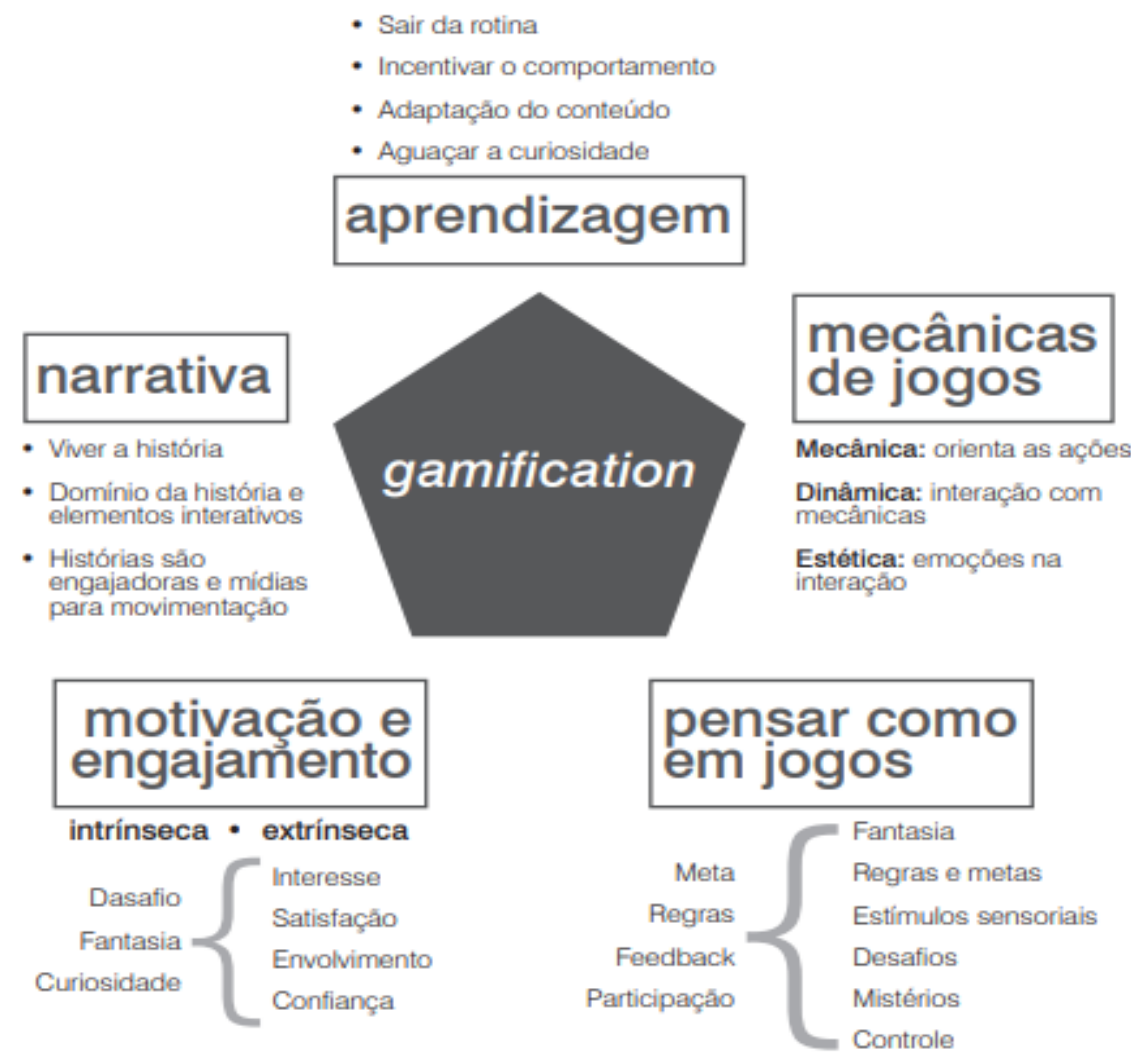

Fonte: Busarello (2016)

A Figura 1 evidencia que a gamificação estimula a aprendizagem, motivação e engajamento, bem como a utilização de uma narrativa, mecânicas de jogos que permitem que os alunos pensem no jogo, e despertem sua curiosidade para favorecer o desenvolvimento do ensino e aprendizagem.

Diante disso, percebe-se que o conceito de gamificação é bastante amplo, visto que é uma metodologia que pode ser utilizada tanto por organizações quanto por escolas, visto que permite utilizar por meio de jogos formas de avaliação e resolução de problemas, bem como a possibilidade de interação entre indivíduos por meio de um ambiente mais interativo.

\subsection{Conceituando jogos}

Os jogos correspondem a uma atividade que possui um ou mais jogadores, procurando conquistar objetivos quantificáveis por meio de um sistema de regras, estes possuem como dimensões específicas o controle, desafio e feedback, que permitem uma maior efetividade da plataforma (SALEN; ZIMMERMAN, 2003). 
A base para a construção de histórias em mídias sequencias ou literárias e de histórias em jogos se traduz na construção de uma narrativa, ou seja, no ato de contar histórias (BUSARELLO, 2016).

Para Mendes (2006) as estratégias dos jogos são como motores motivacionais para os sujeitos, visto que permitem que estes sejam conduzidos ao e podem conduzir ao engajamento em vários aspectos e em diferentes ambientes. Isso mostra que os jogos favorecem o desenvolvimento da aprendizagem.

Em complemento, Vieira e Hoyos (2018, p. 6) explicam que "por meio do jogo, o aprendiz demonstra mais interesse e participa de forma mais autêntica embalado pela afetividade gerada, expressando suas opiniões e desenvolvendo a fala de modo natural e espontâneo".

É importante ressaltar que não se deve confundir gamificação com criação de jogos, pois esta envolve a aplicação de elementos que são usados na construção de jogos eletrônicos (VIANNA, 2013). Por isso, a necessidade de utilizar no ambiente escolar apenas os elementos e mecanismos que estão relacionados aos jogos.

Enfim, os jogos são importantes meios para impulsionar o ensino e aprendizagem, no entanto na gamificação, se utilizam apenas utiliza elementos dos jogos para tornar atividades que são consideradas cansativas, mais atrativas, por isso é necessário conhecer sua aplicação na educação.

\subsection{Gamificação na escola}

A gamificação como estratégia de ensino pode envolver um exemplo de ensino de metodologia ativa em que o professor, por meio de regras claras (contrato didático), estimula os alunos para realizarem missões (tarefas), visando estimular o ensino e aprendizagem (SILVA et al., 2018).

Ainda, consiste na utilização de elementos, não para jogar, mas sim para estimular a motivação, envolvimento nas atividades, proporcionando a aprendizagem, resolução de problemas, e desenvolvimento dos alunos no ambiente escolar (SILVA; SALES, 2017).

Além disso, a gamificação surge como uma proposta educacional de abordagem multimodal, buscando consolidar o processo de aprendizagem, para despertar o interesse e a participação dos indivíduos por meio da utilização de elementos modernos para a execução de tarefas e alcance dos objetivos (ORLANDI, 2018). 
Por sua vez, Zichermann e Cunningham (2011) explicam que esta possibilita uma mudança de comportamento, ou seja, tornando as tarefas consideradas enfadonhas do cotidiano em algo divertido, o que pode é uma importante estratégia pedagógica para melhoria do ensino.

Para Alves, Minho, Diniz (2014, p. 83) “a gamificação surge como uma possibilidade de conectar a escola ao universo dos jovens com o foco na aprendizagem, por meio de práticas como sistemas de rankeamento e fornecimento de recompensas".

Dessa forma, a utilização da gamificação contribui para o desenvolvimento do ensino e aprendizagem dos alunos na escola, possibilitando por meio de jogos a interação e o compartilhamento de ideias entre educadores e alunos, o que permite o estimulo a criatividade e a resolução de problemas.

\subsection{Bibliometria}

A bibliometria busca analisar as atividades científicas ou técnicas por meio de estudos quantitativos das publicações, ou seja, é um método flexível para avaliar a quantidade e a qualidade das fontes de informação, sendo que essa análise gera como produto os indicadores científicos das produções (SILVA; HAYASHI, 2011).

Em complemento, Araújo e Alvarenga (2011) explicam que esta tem um papel importante na análise da produção científica de um país, visto que seus indicadores permitem verificar o grau de desenvolvimento de uma área do conhecimento de um campo científico.

Segundo Pinto e Matias (2011) os indicadores bibliométricos viabilizam a obtenção de uma visão da produção científica e vem sendo utilizados por agências de fomento como um elemento para avaliar a produtividade da comunidade científica.

No entanto, entende-se que a bibliometria pode ser vista e utilizada como estratégia estruturadora de uma pesquisa e não somente como técnica voltada ainvestigação de um estudo já estruturado e fundamentado (MATTOS, 2004).

Segundo Kobashi e Santos (2008) a análise bibliométrica possibilita identificar a quantidade de trabalhos relacionados a um específico assunto, a data de publicação, a publicação por um determinado autor, o grau de desenvolvimento de $\mathrm{P} \& \mathrm{D}$ e de inovação, entre outros. Isso mostra que essa análise favorece a identificação de produções científicas de diferentes assuntos e o desenvolvimento das pesquisas sobre esses assuntos ao longo dos anos. 
Diante disso, percebe-se que a utilização da bibliometria favorece a análise do desenvolvimento das produções científicas, identificando o crescimento de determinada áreas do conhecimento ou temas específicos, o que permite destacar autores e países que estão produzindo esses conteúdos e a evolução da aplicação destes temas ao longo dos anos.

\section{METODOLOGIA}

A pesquisa se caracteriza como exploratória quantitativa, com a utilização de bibliometria. Para Gil (2006, p. 41) explica que a pesquisa exploratória "tem como objetivo proporcionar maior familiaridade com o problema, com vistas a torná-lo mais explícito ou a construir hipóteses".

Este estudo foi classificado como quantitativo, pois foram utilizadas técnicas que produziram dados em forma de números (NEUMAN, 2014). Além disso, foi realizada uma análise bibliométrica de artigos publicados, relacionados à utilização da gamificação na educação. Foi realizada uma busca na base de dados on-line Scopus, visando mapear a produção sobre o tema analisado.

Na base Scopus foram utilizadas as palavras-chave "gamification and education" no campo "ArticleTitle, Abstract, Keywords", sendo aplicado o filtro apenas para selecionar os artigos de acesso livre; foram encontradas 210 publicações de 2013 a 2019.

Os dados encontrados das bases foram exportados para uma planilha e analisados em conjunto, conforme pode ser visualizado na Figura 2.

\section{Figura 2 - Processo de análise dos artigos}

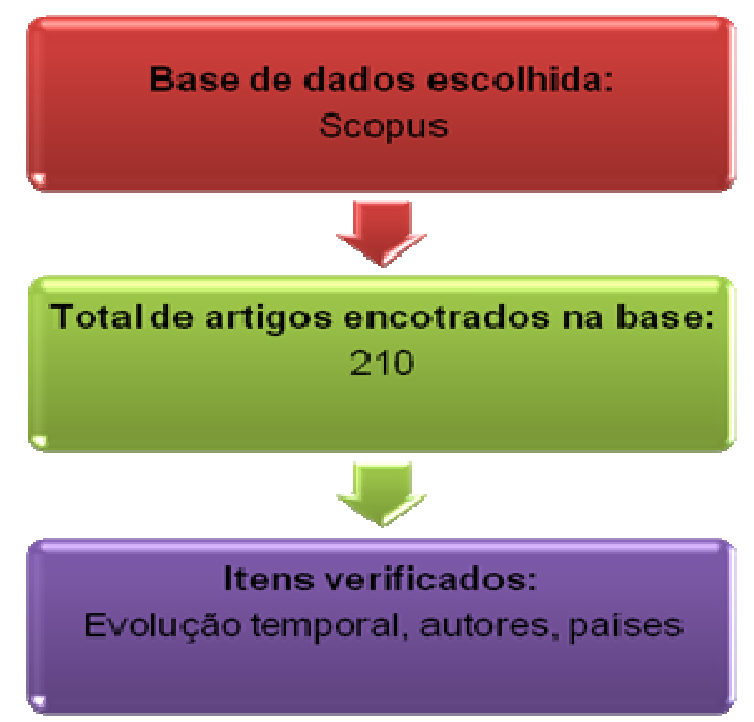

Fonte: Autores (2020). 
Após o levantamento das informações encontradas na busca realizada na base Scopus, os dados foram analisados priorizando a evolução temporal, os autores e os países que mais possuíam publicações sobre o tema em estudo. Ademais, foi utilizado o programa VOSviewer que cria mapas bibliométricos por meio da análise dos artigos encontrados na base Scopus.

\section{RESULTADOS E DISCUSSÕES}

$\mathrm{Na}$ pesquisa realizada na base Scopus, foram analisadas produções científicas relacionadas ao tema gamificação, tendo ocorrido, estas publicações, entre os anos de 2013 a 2019. A Figura 3 mostra a evolução anual dos artigos na base Scopus, destacando que a partir de 2016 houve um crescimento na produção de artigos relacionados à utilização da gamificação na educação.

Figura 3 - Distribuição de artigos por ano (2013 - 2019)

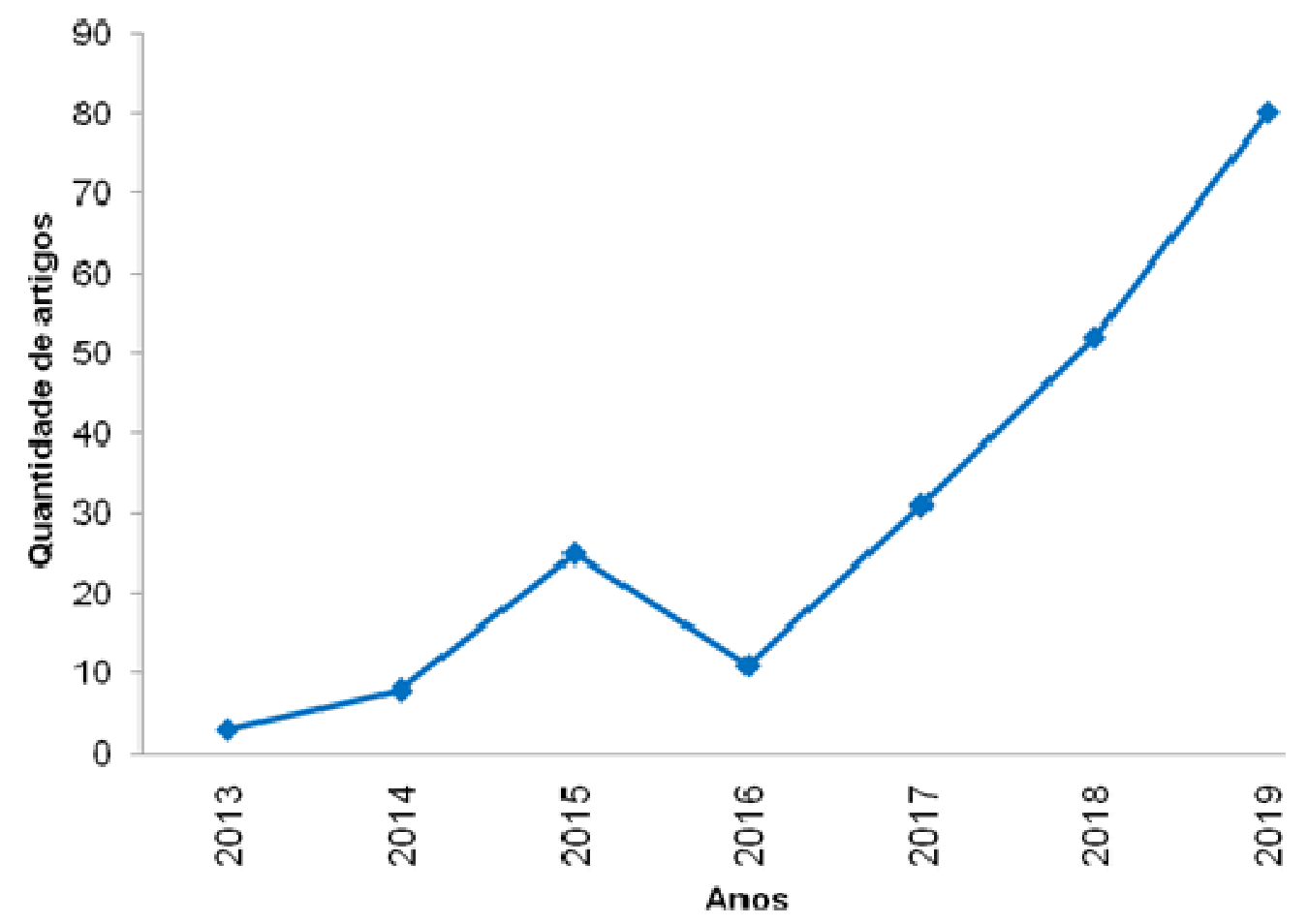

Fonte: Baseado em dados da base Scopus (2020).

Percebe-se na Figura 3, que o ano de 2019 apresentou o maior número de artigos sobre o tema, 80, representando 38\% dos dados encontrados. Analisando o intervalo de 2017 a 2019, no qual houve aumento das publicações, observa-se que foram publicados 163 artigos, 
totalizando $78 \%$ das publicações, mostrando que a produção sobre este tema vem crescendo ao longo dos anos.

Ainda, entende-se que mesmo as publicações sobre gamificação serem recentes, a interação com os games no caso do cenário brasileiro teve início com a chegada do Atari 2600 na década de 1980 (ALVES, MINHO, DINIZ, 2014). E atualmente elementos desses games são utilizados por educadores e empresas para desenvolvimento de atividades mais interativas.

A Figura 4 apresenta os dez autores com maior quantidade de publicações sobre o tema analisado, sendo que Kim, S., Soboleva, E.V., Song, D., Su, C.H., Xu, H. foram os autores que mais publicaram sobre gamificação, com 4 publicações cada.

\section{Figura 4 - Autores com maior frequência de publicações}

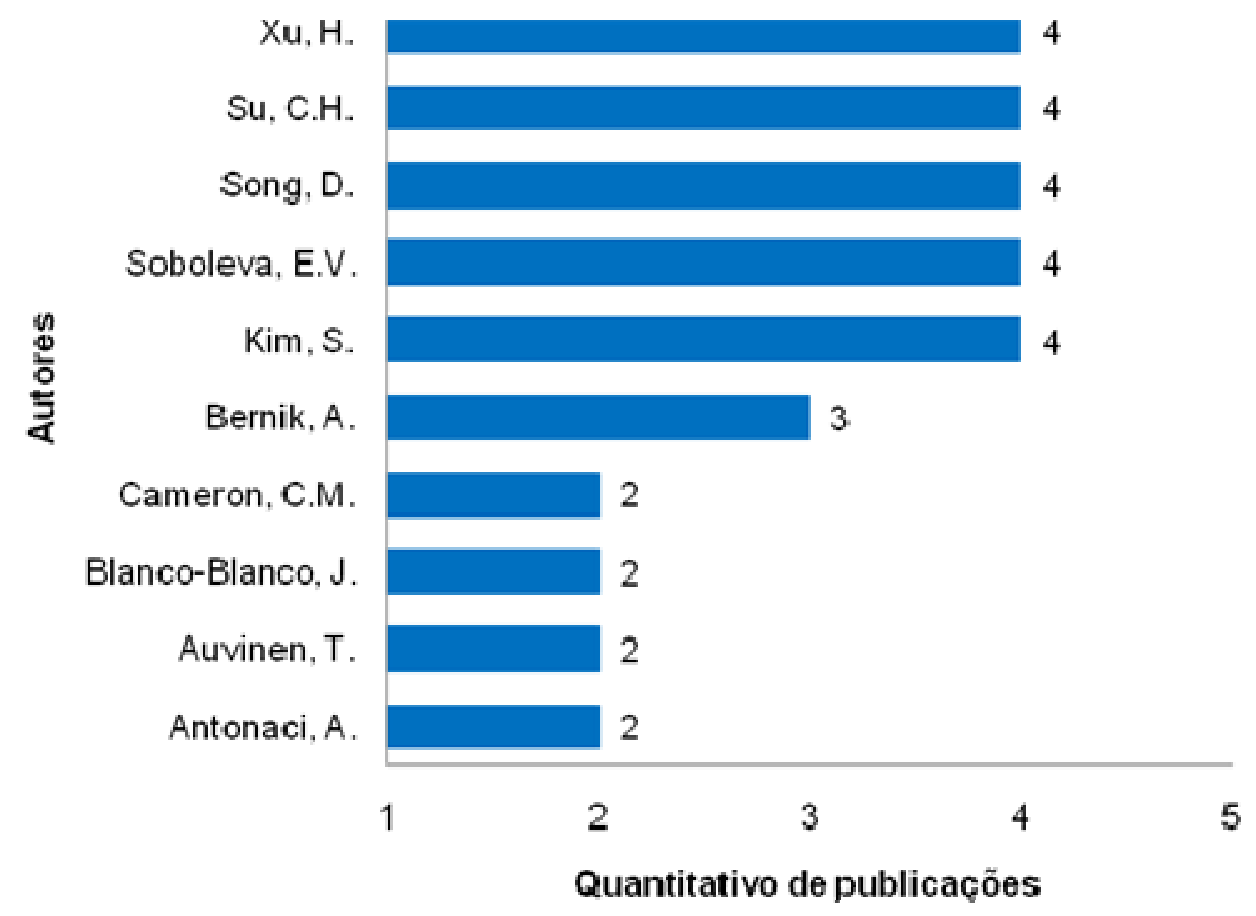

Fonte: Baseado em dados da base Scopus (2020)

A Figura 5 destaca os dez países que há uma concentração maior de publicações relacionadas a gamificação, sendo que o país com maior número de publicações foi a Espanha, com 35 incidências, representando 24\%,; seguida de Estados Unidos com 32 (22\%) e Rússia com 16 (11\%). 
Percebeu-se que o Brasil está presente na relação de países que publicaram sobre o tema, mas não entre os dez países que realizaram mais publicações voltadas a gamificação aplicada à educação, apresentando apenas 5 publicações.

\section{Figura 5 - Países com mais publicações}

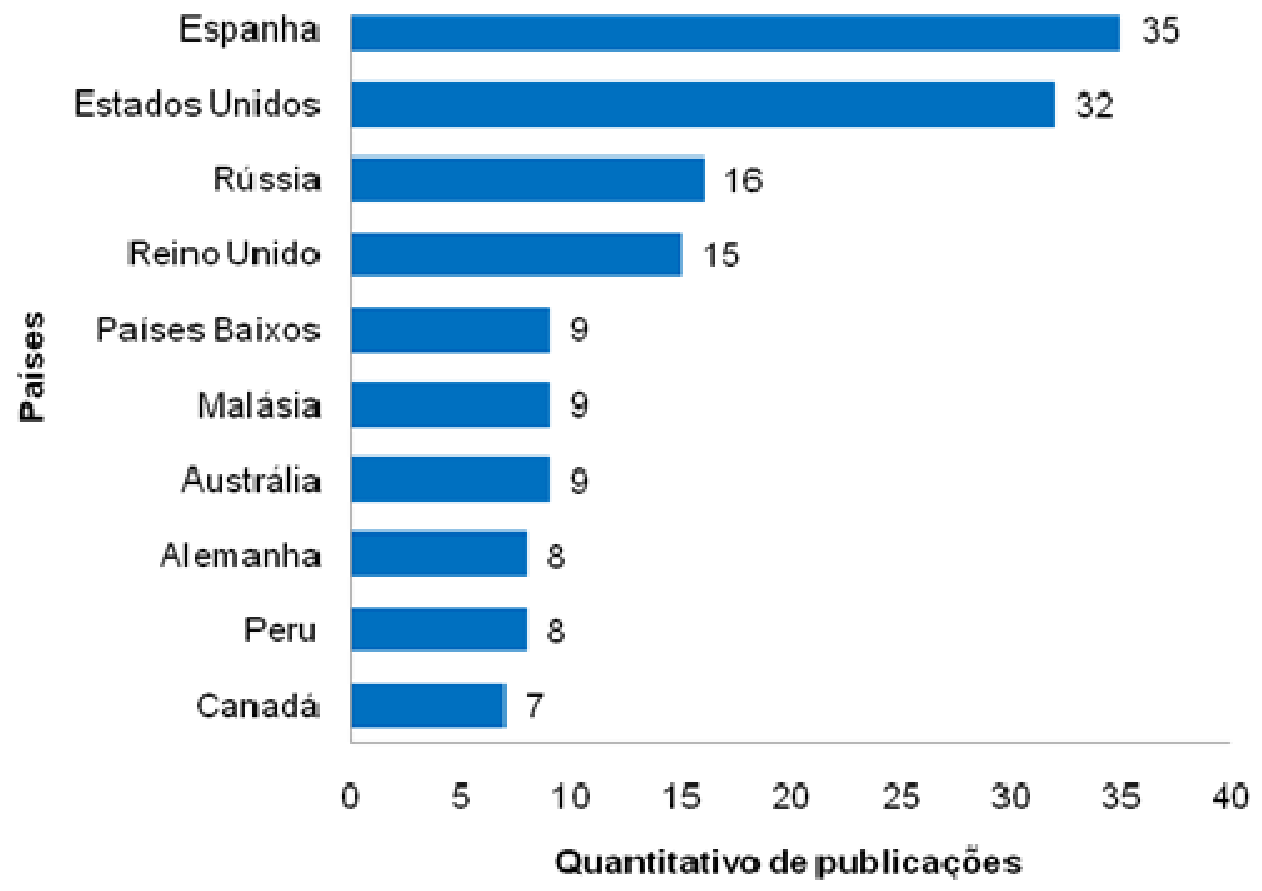

Fonte: Baseado em dados da base Scopus (2020)

A Figura 6 destaca a utilização do VOSviewer, programa de computador que possui acesso livre e é utilizado para criar mapas bibliométricos. Lembrando que neste estudo buscou-se construir uma rede de ocorrências de palavras-chave que foram utilizadas pelos autores dos 210 artigos encontrados no levantamento de dados na base Scopus.

Por meio do VOSviewer, identificou-se a frequência das palavras-chaves, sendo encontradas 54. Deste número, foram retiradas todas as palavras que não estavam direcionadas a proposta deste estudo, sobrando 27 palavras que formaram 5 clusters, que estão representados por diferentes cores, que significam a divisão de aglomerados entre as palavras. 
Figura 6 - Ocorrências de palavras-chave

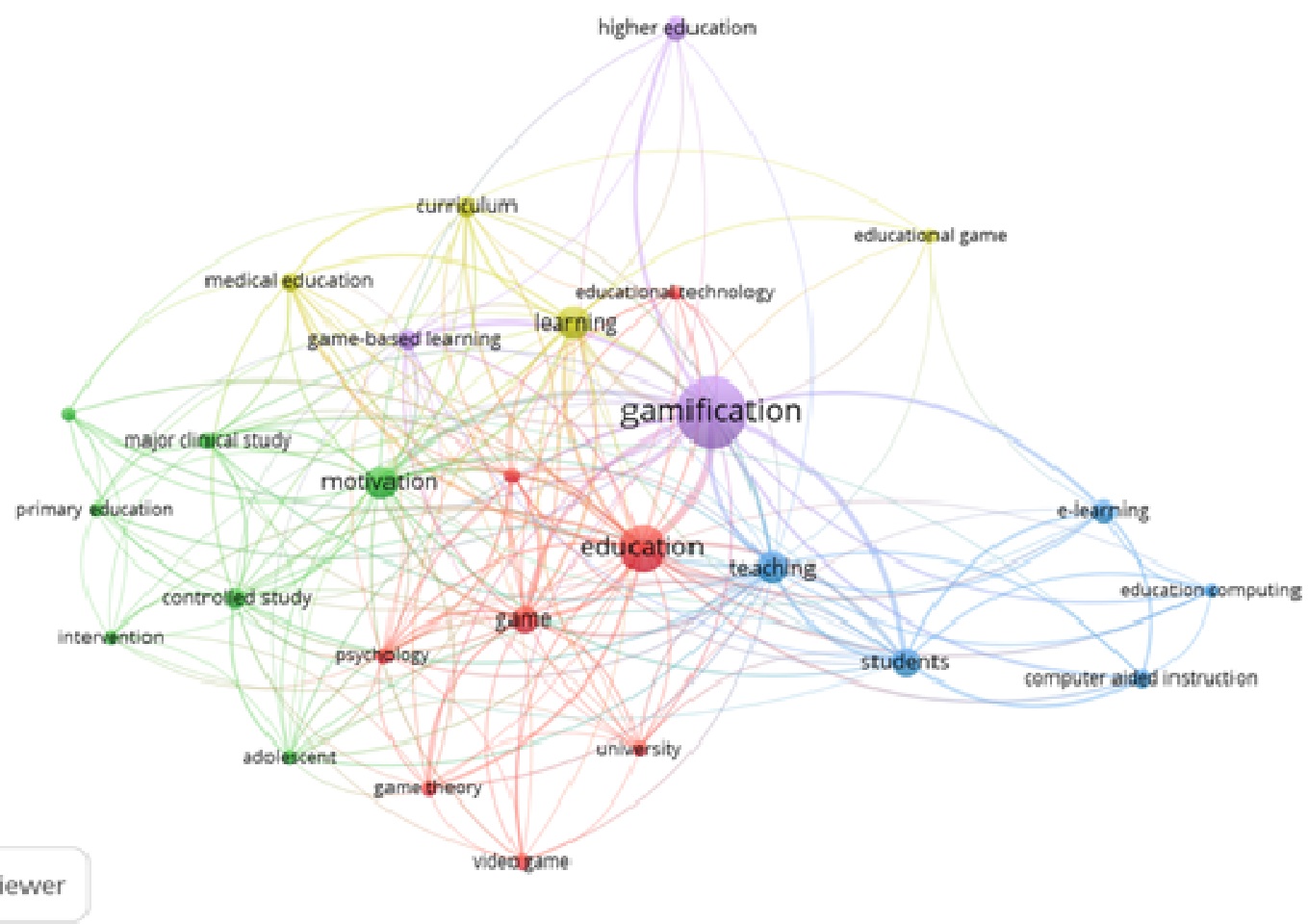

Fonte: VOSViewer (2020)

Percebe-se, analisando os termos, que a maioria das palavras estão relacionadas a aplicação da gamificação à educação, mostrando que o VOSviewer encontrou diferentes palavras voltadas ao tema proposto.

\section{CONSIDERAÇÕES FINAIS}

A pesquisa utilizou a bibliometria visando analisar as publicações relacionadas à aplicação da gamificação na educação; esse tipo de ferramenta permite que sejam identificados artigos sobre determinada área e como está o desenvolvimento desta área de conhecimento a qual esta se analisando.

Nesta pesquisa foi utilizada a base Scopus, visto que abrange um maior número de publicações. Ao analisar as informações encontradas na base verificou-se que as pesquisas sobre gamificação são recentes, visto que os dados são entre 2013 a 2019.

No entanto, percebe-se o quanto essa metodologia vem crescendo no cenário da educação, visto que o número de publicações vem crescendo ao longo dos anos, 
principalmente em 2019, isso evidencia que os educadores estão buscando novas técnicas para melhorar o ensino e aprendizagem dos alunos, como é o caso da utilização da gamificação.

Além disso, identificou-se que existem artigos publicados no Brasil sobre esse tema mais são poucos no caso do levantamento feito por essa base, mais isso não quer dizer que não quer dizer que a utilização dessa metodologia não esteja ocorrendo, está, porém, não com maior ênfase, como ocorre em outros países que investem em metodologias ativas para melhorar a aprendizagem dos alunos no ambiente escolar.

Ainda, por meio da utilização do programa VOSviewer, constatou-se que a maioria das palavras está direcionada a aplicação da gamificação nas escolas, o que mostra que a pesquisa se aprofundou nos artigos de acesso livre que trabalham e discutem esse tema.

Dessa forma, evidencia-se que a utilização da bibliometria possibilita a identificação e análise de variados assuntos, contribuindo assim para ampliação das pesquisas e visando também proporcionar uma análise mais precisa sobre um determinado assunto ou área do conhecimento.

Como sugestão para trabalhos futuros, pode-se analisar a utilização da gamificação também no cenário empresarial, visto que além da aplicação por educadores, esta pode ser aplicada por gestores para melhor o desenvolvimento de atividades e tomada de decisão.

\section{REFERÊNCIAS}

ALVES, L. R. G.; MINHO, M. Diniz. M. Gamificação: diálogos com a educação. In: Luciane Maria Fadel, Vania Ribas Ulbricht, Claudia Regina Batista e Tarcísio Vanzin. (Org.). Gamificação na educação. 1ed.São Paulo: Pimenta Cultural, v. 1, p. 73-95, 2014.

ARAÚJO, R. F.; ALVARENGA, L. A bibliometria na pesquisa científica da pós-graduação brasileira de 1987 a 2007. Encontros Bibli: revista eletrônica de biblioteconomia e ciência da informação, v. 16, n. 31, p.51-70, 2011.

BUSARELLO, R. I. Gamification: princípios e estratégias. São Paulo: Pimenta Cultural, 2016.

DUARTE, G. B. O processo de gamificação e a aprendizagem de línguas pelo viés da Complexidade. In: Congresso Ibero-Americano de Ciência, Tecnologia, Inovação e Educação. Anais... Buenos Aires, 2014, p. 1-14.

GARRIS, R.; AHLERS, R.; DRISKELL, J. E. Games, motivation, and learning: A research and practice model. Simulation \& Gaming, v. 33, n. 4, p. 441-467, 2002. 
GIL, A. C. Como elaborar projetos de pesquisa. 4. ed. 8. reimpr. São Paulo: Atlas, 2006.

KAPP, K. M. The gamification of learning an dinstruction: game-based methods and strategies for training and education. São Francisco: Pfeifer/ASTD, 2012.

KOBASHI, N. Y.; SANTOS, R. N. M. ARQUEOLOGIA DO TRABALHO IMATERIAL: ma aplicação bibliométrica à análise de dissertações e teses. Encontros Bibli: revista eletrônica de biblioteconomia e ciência da informação, n. esp., $1^{\circ}$ sem. 2008.

LEFFA, V. J.; PINTO, C. M. Aprendizagem como vício: o uso de games na sala de aula. Revista (Con)Textos Linguísticos, v.8, n. 10.1, p. 358-378, 2014.

MATTOS, P. L. C. L. de. "Bibliometria": a metodologia acadêmica convencional em questão. RAE eletrônica, v. 3, n. 2, p. 01-06, 2004.

MENDES, E. G. A radicalização do debate sobre inclusão escolar no Brasil. Revista Brasileira de Educação, v. 11, n. 33, p. 387-405, 2006.

NEUMAN, W. L. Social Research Methods: Qualitative and Quantitative Approaches. Seventh edition, Pearson New International Edition. Harlow Pearson Education Limited, 2014.

ORLANDI, T. R. C et al. Gamificação: uma nova abordagem multimodal para a educação. Biblios, n. 70, p. 17-30, 2018.

PANTOJA, A. S.; PEREIRA, L. M. Gamificação: como jogos e tecnologias podem ajudar no ensino de idiomas. Estudo de caso: uma escola pública do Estado do Amapá. Estação Científica (UNIFAP), v. 8, n. 1, p. 111-120, 2018.

PINTO, A. L; MATIAS, M. Indicadores Científicos e as Universidades Brasileiras. Londrina, v. 16 n. 3, p. 1- 18., jan./ jun. 2011.

RODRIGUES, J. J. C. A GAMIFICAÇÃO COMO ESTRATÉGIA PARA O ENSINO: Um Estudo sobre as Aulas de Língua Inglesa em uma Escola Pública. In: International Congress of Critical Applied Linguistics. Anais... Brasília, p. 948 -967, 2015.

SAlAMI, A. D. G.; SILVA, E. R.; TISOTT, P. B.; DIAS, D. T. A. D.; BOCCHESE, P. Gamificação: a Proposição de um Modelo para Aplicação nas Empresas. In: XVIII Mostra de Iniciação Científica, Pós-Graduação, Pesquisa e Extensão, Programa de Pós Graduação em Administraçaõ - UCS, 2018.

SALEN, K; ZIMMERMAN, E. Rulesof play: Game design fundamentals. Cambridge, MA: MIT Press, 2003.

SILVA, J. B et al. Tecnologias digitais e metodologias ativas na escola: o contributo do Kahoot para gamificar a sala de aula. Revista Thema, v. 15, n. 2, p. 780-791. 2018.

SILVA, J. B.; SALES, G. L. Gamificação aplicada no ensino de Física: um estudo de caso no ensino de óptica geométrica. Acta Scientiae, v.19, n. 5, p.782-798, 2017. 
SILVA, J. B.; SALES, G. L.; CASTRO, J. B. Gamificação como estratégia de aprendizagem ativa no ensino de Física. Revista Brasileira de Ensino de Física, v. 41, n. 4, 2018.

SILVA, M. R.; HAYASHI, C. R. M; HAYASHI, M. C. P. I. Análise bibliométrica e cientométrica: desafios para especialistas que atuam no campo. InCID: Revista de Ciência da Informação e Documentação, v. 2, n. 1, p. 110-129, jan./jun. 2011.

VIANNA, Y.; VIANNA, M.; MEDINA, B.; TANAKA, S. Gamification, Inc.: Como reinventar empresas a partir de jogos. MJV Press: Rio de Janeiro, 2013.

VIANNA, Y. Gamification, Inc: como reinventar empresas a partir de jogos. $1^{\text {a }}$ Ed. Rio de Janeiro: MJV Press, 2013.

VIEIRA, M. F.; HOYOS, E. A. C. Gamificação no Ensino das Línguas Portugues e Espanhola: relato de experiência no IFSP Avaré. Revista CBTecLE, v. 1, n.2, 2018.

ZICHERMANN, G.; CUNNINGHAM, C. Gamification by design: implementing game mechanics in web and mobile apps. Sebastopol, CA: O’ Reilly Media, Inc. 2011.

\section{Como Referenciar este Artigo, conforme ABNT:}

CRUZ, C. A. B; NEJAIM, V. M; BEZERRA, A. S. S. Gamificação como Estratégia para Estimular a Aprendizagem dos Alunos na Escola: Uma Análise Bibliométrica. Rev. FSA, Teresina, v.18, n. 02, art. 10, p. 183-197, fev. 2021.

\begin{tabular}{|l|c|c|c|}
\hline \multicolumn{1}{|c|}{ Contribuição dos Autores } & $\begin{array}{c}\text { C. A. B. } \\
\text { Cruz }\end{array}$ & $\begin{array}{c}\text { V. M. } \\
\text { Nejaim }\end{array}$ & $\begin{array}{c}\text { A. S. S. } \\
\text { Bezerra }\end{array}$ \\
\hline 1) concepção e planejamento. & $\mathrm{X}$ & & \\
\hline 2) análise e interpretação dos dados. & $\mathrm{X}$ & $\mathrm{X}$ & $\mathrm{X}$ \\
\hline 3) elaboração do rascunho ou na revisão crítica do conteúdo. & $\mathrm{X}$ & $\mathrm{X}$ & $\mathrm{X}$ \\
\hline 4) participação na aprovação da versão final do manuscrito. & $\mathrm{X}$ & $\mathrm{X}$ & $\mathrm{X}$ \\
\hline
\end{tabular}

